\title{
Die niederländische Irrenanstaltspflege in den Jahren 1875 bis 1900 .
}

\author{
Von \\ Dr. D. Schermers, \\ ärztlicher Direktor des Chr. Sanatoriums für Nervenkranke in Zeist bel Utrecht. \\ Mit 6 Textfiguren.
}

(Eingegangen am 10. August 1910.)

In den letzten Jahren wurde in der psychiatrischen Welt in allem Ernste die Frage erwogen, ob die Resultate der Anstaltspflege von Geisteskranken wohl in richtigem Verhältnis stehen zu den enormen damit verbundenen Ausgaben. Es wurde darauf hingewiesen, daß kostspielige Anstalten gebaut sind, $d a ß$ die Anforderungen auf hygienischem Gebiet stets höher werden, daß auch die Pflege ohne Zweifel viel besser wird und daß trotzdem die Resultate der Pflege nicht günstiger sind. Man hat selbst die Meinung ausgesprochen, daß es unmöglich ist, einen Zusammenhang zwischen ärztlicher Behandlung in einer Anstalt und Genesung zu entdecken. Wo Heilung erwartet werden kann, wird diese bei den internierten Kranken nicht schneller vor sich gehen, als dies unter anderen Umständen der Fall sein würde.

Es versteht sich von selbst, daß diese Anschauungen einen tiefen Eindruck auf die psychiatrische Welt gemacht haben und solche pessimistische Bemerkungen von verschiedenen Seiten ernstlich bekämpft sind.

Es ist jedoch sehr fraglich, ob es überhaupt möglich ist, einen Vergleich zu treffen zwischen Geisteskranken, die in einer Anstalt und solchen, die außerhalb derselben gepflegt werden. Bei den Resultaten der Pflege hat man mit zahllosen unbekannten Faktoren zu tun, wodurch eine Vergleichung, wenigstens eine richtige, einfach unmöglich ist. Hierzu wäre es nötig, daß man es mit ganz gleichartigen Erscheinungen in und außerhalb der Anstalt zu tun hätte, von denen die Prognose mit einiger Sicherheit gestellt werden kann, und außerdem müßte ein und derselbe Beobachter über den Verlauf urteilen, denn, obgleich das Endresultat dasselbe sein wird, so kann dennoch die Krankheit in einem Fall einen viel ernsteren Verlauf nehmen als in einem anderen.

Dessenungeachtet bleibt es immerhin eine sehr wichtige Frage, die sicher eine ernste Erwägung verdient, wenigstens insofern eine Antwort 
darauf möglich ist. Zunächst wünsche ich diese Frage einigermaßen anders zu formulieren und zu versuchen, den Nachweis zu liefern, inwiefern die Ergebnisse der Anstaltspflege jetzt besser sind als früher.

Ich habe diesbezüglich einige Studien gemacht mit Bezug auf die Zahl der Kranken, die in den Jahren 1875-1900 in den niederländischen Irrenanstalten gepflegt wurden. Ich habe berechnet, wie viele Patienten jedes Jahr aufgenommen wurden, wie viele als geheilt entlassen und wie viele verstorben sind. Weiter habe ich noch einzelne Besonderheiten beachtet mit Berücksichtigung des Alters der Kranken, der Rückkehr und der Dauer der Geisteskrankheit, während ich auch die Dauer der Pflege nicht unberücksichtigt ließ; auch habe ich die Art der Psychosen vermerkt. Natürlich konnte ich nicht auf alle Einzelheiten näher eingehen; ich habe nur die Aufmerksamkeit gerichtet auf die Punkte, die von mehr allgemeinem Interesse sind.

\section{Die Aufnahmen.}

Die Zahl der Kranken, welche jedes Jahr in die Irrenanstalten aufgenommen wurden, hat sich in der letzten Hälfte des vorigen Jahrhunderts bedeutend vergrößert. Im Durchschnitt war diese Zahl im letzten Dezennium fast zweiundeinhalbmal so groß als im ersten, wie aus folgender Tabelle zu ersehen ist.

Tabelle 1. Übersicht der durchschnittlichen Aufnahmen.

\begin{tabular}{c|c|c|c}
\hline & Männer & Frauen & Gesamt \\
\hline $1850-1860$ & 300 & $=-1=3$ & 603 \\
$1860-1870$ & 432 & 435 & 867 \\
$1870-1880$ & 524 & 514 & 1037 \\
$1880-1890$ & 605 & 603 & 1208 \\
$1890-1900$ & 789 & 756 & 1545
\end{tabular}

Sehr wahrscheinlich hat die Einführung des neuen Irrengesetzes im Jahre 1884 dabei großen Einfluß ausgeübt. Die Zahl ist bei beiden Geschlechtern merkwürdig dieselbe geblieben; nur im letzten Dezennium war sie bei den Männern etwas größer, aber der Unterschied war doch ganz unerheblich. Das Zahlenmaterial ist den offiziellen Jahresberichten entnommen, welche aus den Jahren 1850-1900 von der staatlichen Behörde vorliegen; die Kranken, welche nur aus einer Anstalt in die andere übergingen, blieben dabei unberücksichtigt.

Es ist die Absicht einiges, das für die Prognose wichtig sein könnte, jetzt etwas mehr hervorzuheben. Die Angaben dafür könnte ich jedoch nicht von der ganzen Periode benützen, nur von den letzten 25 Jahren. Die Zahl der Kranken, welche in diesem Zeitraum von $1875-1900$ in 
die Anstalten aufgenommen wurden, betrug nicht weniger als 37448 , wovon 18978 Männer und 18470 Frauen. Bei diesen Zahlen war es jedoch nicht möglich festzustellen, welche Kranken von der einen Anstalt in die andere versetzt waren, weshalb diese in der Gesamtzahl mitberechnet sind.

Alter. Allgemein wird angenommen, daß das Alter oft einen großen Einfluß hat auf den Verlauf der Geisteskrankheit; deshalb soll zunächst festgestellt werden, wie sich das Verhältnis gestaltet, wenn die Kranken nach dem Alter eingereiht werden. Die folgende Tabelle gibt eine Utbersicht der Kranken, die in jedem Quinquennium aufgenommen wurden, mit Angabe des Alters; zugleich wird angegeben, welches das Verhältnis war mit Bezug auf die ganze Zahl, die in derselben Periode aufgenommen wurden.

Tabelle 2. Alter der aufgenommenen Kranken.

\begin{tabular}{|c|c|c|c|c|c|c|c|c|c|c|c|c|c|c|c|c|c|c|c|c|}
\hline & \multicolumn{3}{|c|}{$1875-1880$} & \multicolumn{3}{|c|}{$1880-1885$} & \multicolumn{3}{|c|}{$1885-1890$} & \multicolumn{3}{|c|}{$1890-1895$} & \multicolumn{3}{|c|}{$1895-1900$} & \multicolumn{3}{|c|}{$1875-1900$} \\
\hline & & & M. & Fr. & Tot. & M. & Fr. & Tot. & M. & Fr. & Tot. & M. & Fr. & Tot. & M. & Fr. & Tot. & M. & Fr. & Tot. \\
\hline \multirow[t]{2}{*}{$0-10$} & & & 33 & 21 & 54 & 24 & 9 & 33 & 43 & 34 & 77 & 66 & 51 & 117 & 115 & 22 & 187 & 281 & 167 & $4+8$ \\
\hline & & Rel. & 1,2 & 0,8 & 0,9 & 0,8 & 0,3 & 0,5 & 1,1 & 0,8 & 0,9 & 1,6 & 1,2 & $1, t$ & 2,2 & 1,0 & 1,6 & 1,5 & 0,9 & 1,2 \\
\hline \multirow[t]{2}{*}{$10-20$} & ", & Abs. & 196 & 139 & 335 & 159 & 123 & 282 & 294 & 198 & 442 & 363 & 267 & 630 & 487. & 254 & 741 & 1449 & 981 & 2430 \\
\hline & & Rel. & 6,9 & 5,1 & 6,0 & 5,5 & 4,5 & 5,0 & 6,3 & 4,9 & 5,6 & 8,6 & 6,6 & 7,7 & 9,3 & $\tilde{5}, 1$ & 7,2 & 7,6 & 5,3 & 6,5 \\
\hline \multirow[t]{2}{*}{$20-30$} & $"$ & Abs. & 615 & 579 & 1194 & 656 & 530 & 1186 & 868 & 810 & 1678 & 905 & 853 & 1758 & 1132 & 1033 & 2167 & 4176 & 3807 & 7983 \\
\hline & & Rel. & 21,8 & 21,3 & 21,6 & 22,8 & 19,4 & 21,1 & 22,4 & 20,3 & 21,3 & 21,6 & 21,1 & 21,4 & 21,7 & 20,8 & 21,2 & 22,0 & 20,6 & 21,3 \\
\hline \multirow[t]{2}{*}{$30-40$} & $"$ & Abs. & 685 & 613 & 1298 & 711 & 658 & 1369 & 896 & 1029 & 1925 & 864 & 874 & 1738 & 1189 & 1120 & 2309 & 4345 & 4294 & 8630 \\
\hline & & Rel. & 24,3 & 22,5 & 23,4 & 24,7 & 24,0 & 24,3 & 23,1 & 25,8 & 24,4 & 20,6 & 21,6 & 21,7 & 22,7 & 22,5 & 22,6 & 22,9 & 23,2 & 23,1 \\
\hline \multirow[t]{2}{*}{$40-50$} & $"$ & Abs. & 561 & 574 & 1135 & 582 & 573 & 1155 & 739 & 729 & 1468 & 732 & 746 & 1478 & 989 & 926 & 1915 & 3603 & 3548 & 7151 \\
\hline & & Rel. & 10,9 & $\mid 21,1$ & 20,5 & 20,2 & 20,9 & 20,5 & 19,0 & 18,3 & 18,6 & 17,5 & 18,5 & 18,0 & 18,9 & 18,6 & 18,7 & 19,0 & 19,2 & 19,1 \\
\hline \multirow[t]{2}{*}{$50-60$} & " & Abs. & 398 & 402 & 800 & 428 & 428 & 856 & 597 & 585 & 1182 & 632 & 576 & 1208 & 598 & 692 & 1290 & 2653 & 2683 & 5336 \\
\hline & & Rel. & 14,1 & 14,8 & 14,4 & 14,8 & 15,6 & 15,2 & 15,4 & 14,6 & 15,0 & 15,1 & 14,2 & 14,7 & 11,4 & 13,9 & 12,6 & 14,0 & 14,5 & 14,2 \\
\hline \multirow[t]{2}{*}{$60-70$} & ", & Abs. & 224 & 244 & 468 & 213 & 260 & 473 & 351 & 416 & 767 & 410 & 397 & 807 & 434 & 517 & 951 & 1632 & 1834 & 3466 \\
\hline & & Rel. & 7,9 & 8,9 & 8,4 & 7,4 & 9,5 & 8,4 & 9,0 & 10,4 & 9,7 & 9,8 & 9,8 & 9,8 & 8,3 & 10,3 & 9,3 & 8,6 & 9,9 & 9,2 \\
\hline \multirow[t]{2}{*}{ über 70} & , & Abs. & 99 & 148 & 247 & 105 & 155 & 260 & 140 & 192 & 332 & 212 & 266 & 478 & 279 & 379 & 658 & 835 & 1140 & 1975 \\
\hline & & Ret. & 3,5 & 5,4 & 4,4 & 3,6 & 5,6 & 4,6 & 3,6 & 4,8 & 4,2 & 5,0 & $\mid 6,6$ & 6,8 & 5,3 & 7,4 & 6,4 & 4,4 & 6,1 & 5,3 \\
\hline
\end{tabular}

Es fragt sich jetzt, ob bei den Aufgenommenen ein merkbarer Unterschied im Alter wahrzunehmen ist, ob heute ein bestimmtes Lebensalter mehr bei ihnen zu beobachten ist als früher. Ist es möglich, daß dadurch ein günstiger oder ungünstiger Einfluß auf den Verlauf der Geisteskrankheit ausgeübt wurde?

Wenn man die Verhältnisse in jedem Quinquennium in dieser Hinsicht miteinander vergleicht, so findet man wohl einige kleine Differenzen, aber wie hätte man anderes erwarten können? Es stellte sich z. B. heraus, daß die Zahl der Kranken jünger als 20 Jahre etwas zugenommen hat, daß das Verhältnis im Alter von 20-30 Jahren ziem- 
lich dasselbe blieb, die Gesamtziffer im späteren Alter, wenn auch nur um ein weniges, sich verminderte und im Alter von 60 Jahren und darüber wieder ein wenig stieg. Die Unterschiede waren jedoch so gering und außerdem so schwankend, daß kein bestimmter Schluß daraus gezogen werden darf.

Man kann also nicht behaupten, daß die Kranken jetzt im ganzen jünger oder älter in die Anstalten aufgenommen werden als früher; in dem Altersunterschied kann also kein deutlich hervortretender Faktor gefunden werden, wodurch der Verlauf der Geisteskrankheit sich jetzt günstiger oder ungünstiger als früher gestaltet hat.

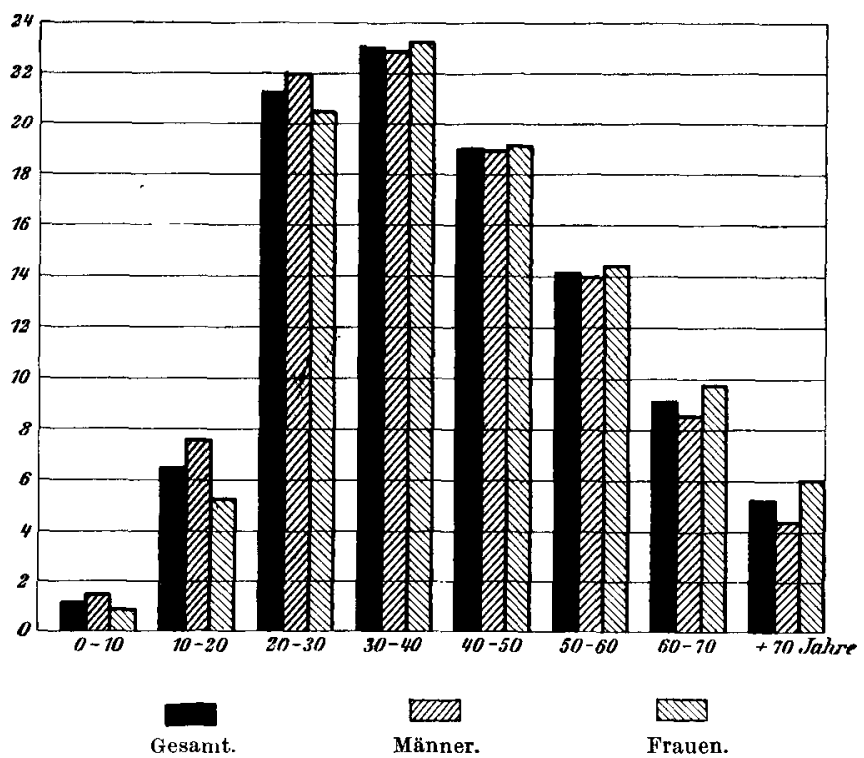

Fig. 1. Verteilung der Aufnahmen auf die einzelnen Altersstufen. (37448 Patienten; 18978 Männer, 18470 Frauen.)

Aus der Tabelle folgen weiter noch einige allgemeine Angaben über das Alter derjenigen, die in eine Irrenanstalt aufgenommen sind, wovon die Fig. 1 eine nähere Übersicht gibt. Es wird dabei angegeben, wie die Kranken sich verteilt haben nach dem Lebensalter während der ganzen Periode. Fs ergibt sich daraus, daß die meisten Kranken, die in die Irrenanstalt aufgenommen wurden, ein Lebensalter hatten von 30-40 Jahren; die Zahl derer, die 20-30 Jahre alt waren, war jedoch nur um ein weniges geringer. Nach dem 40. Jahre wurde das Verhältnis allmählich geringer; jünger als 10 Jahre wurden nur sehr wenige Kranke aufgenommen.

Bezüglich des Geschlechts kann aufgemerkt werden, daß unter 30 Jahren mehr männliche Kranke aufgenommen wurden, während nach 60 Jahren die Zahl bei den weiblichen bedeutend größer wird. 
Rückkehr. Ein anderer Faktor, der für die Prognose von Bedeutung sein könnte, ist die Rückkehr der Geisteskrankheit; eine Ubersicht, wie viele Male die Patienten schon krank waren, bevor sie in die Anstalt aufgenommen wurden, findet man in Tabelle 3, wo man auch die Verhältnisse angegeben findet auf $100 \mathrm{Kranke,} \mathrm{die} \mathrm{in} \mathrm{derselben} \mathrm{Zeit} \mathrm{auf-}$ genommen wurden.

Tabelle 3. Rückkehr der Geisteskrankheit.

\begin{tabular}{|c|c|c|c|c|c|c|c|c|c|c|c|c|c|c|c|c|c|c|}
\hline & & & $75-1880$ & 188 & $30-18$ & 885 & & $35-18$ & 890 & & $90-18$ & 895 & 189 & $5-19$ & 900 & & $75-19$ & 900 \\
\hline & & M. & Fr. Tot. & M. & Fr. & | Tot. & M. & Fr. 1 & Tot. & M. & Fr. & |Tot. & M. & Fr. & Tot. & M. & Fr. & | Tot. \\
\hline \multirow[t]{2}{*}{ Erstes Mal } & & 190 & 20594249 & 2292 & 2042 & 4334 & 2114 & 31256 & 6239 & 3488 & $|3232| \mathrm{C}$ & 26720 & 4333 & 40198 & 8352 & 154171 & 14477 & 2989 \\
\hline & Rel. & 77,8 & $75,7: 76,8$ & $79,6 \mid$ & 74,6 & 77,2 & 80,3 & 78,3 & 79,2 & 83,3 & 80,1 & $1 \mid 81,7$ & 82,9 & 80,6 & 81,8 & 81,2 & 78,4 & 79,2 \\
\hline \multirow[t]{2}{*}{ Zweites Mal } & Abs. & 395 & $377^{\prime} \quad 772$ & 381 & 427 & 808 & 487 & 5541 & 1041 & 467 & 499 & 966 & 535 & 5881 & 1123 & 2265 & $2+45$ & 4711 \\
\hline & Rel. & 14,0 & $13,8,13,9$ & 13,2 & 15,6 & 14,4 & 12,5 & 13,8 & 13,2 & 11,2 & 12,3 & 11,7 & 10,2 & 11,8 & 11,0 & 11,9 & 13,2 & $12, t$ \\
\hline \multirow[t]{2}{*}{ Drittes Mal } & Abs. & 126 & $137 \mid 263$ & 129 & 131 & 260 & 157 & 145 & 302 & 110 & 133 & 243 & 150 & 183 & 333 & 672 & 729 & 1401 \\
\hline & Rel. & 4,5 & \begin{tabular}{ll|l}
5,0 & 4,7
\end{tabular} & 4,5 & 4,8 & 4,6 & 4,0 & 3,6 & 3,8 & 2,6 & 3,3 & $\begin{array}{l}3 \\
3\end{array}$ & 2,9 & 3,7 & 3,2 & 3,5 & 3,9 & 3,2 \\
\hline \multirow{2}{*}{$\begin{array}{l}\text { Mehr als drei- } \\
\text { mal }\end{array}$} & Abs. & 100 & $147 \quad 247$ & 76 & 136 & 212 & 120 & 168 & 288 & 108 & 143 & 3251 & 172 & 181 & 353 & 576 & 775 & $135:$ \\
\hline & kiel. & 3,5 & $5.4 \quad 4,4$ & 2,6 & 4.9 & 3,8 & 3,1 & 4,2 & 3,6 & 2,6 & 3,5 & 53,0 & 3,3 & 3,6 & 3,4 & 3,0 & 4,2 & $3, t$ \\
\hline Unbekannt & & -- &,-- & -1 & $\cdots$ & - & 1 & 1 & 2 & 12 & 29 & 41 & 36 & 14 & 47 & 46 & 14 & $\theta$ \\
\hline
\end{tabular}

Es zeigt sich daraus, daß im ganzen fast $4 / 5$ aller Patienten zum erstenmal geisteskrank waren, als sie in die Anstalt aufgenommen wurden, und $1 / 8$ zum zweitenmal. Von den übrigen waren $3,8 \%$ das dritte Mal krank und 3,6\% mehr als dreimal.

Die Frage ist jetzt, ob in diesen Verhältnissen eine Veränderung stattgefunden hat. Die Antwort muß da bejahend sein; die Zahl derer, die zum erstenmal geisteskrank waren bei ihrer Aufnahme, wird verhältnismäßig größer. Wenn man sich auf die Zahlen stützt, findet sogar eine regelmäßige Zunahme statt. Ist dies ein günstiges oder ein ungünstiges Zeichen?

Im allgemeinen wird es schwer sein, hier eine bestimmte Antwort zu geben; dennoch wird meistens angenommen, daß die Prognose schlechter wird, je nachdem die Geisteskrankheit mehrmals vorkam; also könnte man erwarten, daß die Aussicht auf Genesung jetzt etwas größer sein könnte.

Was das Geschlecht betrifft, stellt sich heraus, daß die Geisteskrankheit bei dem Manne etwas weniger Neigung hat, zurückzukehren, als bei dem Weibe; der Unterschied war jedoch nicht sehr bedeutend.

Dauer. Weiter sollte berücksichtigt werden die Dauer der Geisteskrankheit, bevor der Kranke in die Anstalt aufgenommen wurde. In den offiziellen Berichten wird vermeldet, ob die Krankheit weniger als 3 Monate, 3-12 Monate oder schon länger als ein Jahr bestand; auch 
wird berichtet, ob die Krankheit schon seit der ersten Jugend anwesend war oder ob die Dauer unbekannt war; Tabelle 4 gibt eine UUbersicht hiervon.

Tabelle 4. Dauer der Geisteskrankheit.

\begin{tabular}{|c|c|c|c|c|c|c|c|c|c|c|c|c|c|c|c|c|c|}
\hline & \multicolumn{2}{|c|}{$1875-1880$} & \multicolumn{3}{|c|}{$1880-1885$} & \multicolumn{3}{|c|}{$1885-1890$} & \multicolumn{3}{|c|}{$1890-1895$} & \multicolumn{2}{|c|}{$1895-1900$} & \multicolumn{3}{|c|}{$1875-1900$} \\
\hline & & M. & Fr. Tot. & M. & Fr. & Tot. & M. & Fr. & Tot. & M. & Fr. & Tot. & M. & Fr. 'Tot. & M. & Fr. & Tot. \\
\hline \multirow[t]{2}{*}{ (1)- 3 Monate } & bs. & 982 & 10101992 & 1050 & 1070 & 2120 & 1098 & 1251 & 2349 & 1403 & 1371 & 2774 & 1760 & 1656,3416 & 6293 & 6358 & $1 \leq 651$ \\
\hline & Rel. & 34,9 & $37,1^{\prime} 36,0$ & 36,4 & 39,1 & 37,7 & 28,3 & 31,3 & 29,8 & $33,5 \mid$ & 33,9 & 933,7 & 33,7 & $33,2,33,4$ & 33,2 & 34,4 & 33,8 \\
\hline \multirow[t]{2}{*}{$3--12$} & Abs. & 779 & 7441523 & 668 & 710 & 1378 & 851 & 844 & 1695 & 880 & 901 & 1781 & 1054 & 10202074 & 4232 & 4219 & 8451 \\
\hline & Rel. & 27,7 & $27,32 \pi, 5$ & 23,2 & 25,9 & 24,5 & 21,9 & 21,1 & 21,5 & $21,0 \mid$ & 22,3 & $3: 21,6$ & 20,2 & 20,3 & 22,3 & 22,8 & 22,5 \\
\hline \multirow{2}{*}{$\begin{array}{l}\text { Länger als } \\
\qquad 1 \mathrm{Jahr}\end{array}$} & Abs. & 781 & 7531534 & 8.7 & 749 & 1596 & 1352 & 1323 & 2675 & 1279 & 1340 & 2619 & 1666 & 18263492 & 5925 & 5991 & 11016 \\
\hline & Rel. & 27,7 & $27,6,27,7$ & 29,4 & 27,4 & 28,4 & 34,8 & 33,1 & 33,9 & 30,5 & 33,2 & 31,8 & 31,9 & $36,6,34,2$ & 31,2 & 32,4 & 31,8 \\
\hline \multirow{2}{*}{$\begin{array}{l}\text { Seit der ersten } \\
\text { Jugend }\end{array}$} & Abs. & 204 & $153 \quad 357$ & 214 & 141 & 355 & 351 & 337 & 688 & 423 & 280 & 705 & 552 & 321.873 & 1744 & 1232 & 5976 \\
\hline & Rel. & 7,2 & $5,6 \quad 6,4$ & $\pi, 4$ & 5,1 & 6,3 & 9,0 & 8,4 & 8,7 & 10,1 & 6,9 & 8,5 & 10,5 & $6,4 \mid 8,5$ & 9,2 & 6,7 & 7,9 \\
\hline \multirow[t]{2}{*}{ Lnbekannt } & Abs. & 66 & 60126 & 100 & 66 & 166 & 227 & 238 & 465 & 200 & 144 & \begin{tabular}{l|l|}
4 & 344
\end{tabular} & 191 & $162 \quad 353$ & 784 & 670 & 1454 \\
\hline & Rel. & 2,3 & $|2,2| 2,3 \mid$ & 3,5 & 2,4 & 2,9 & 5,8 & 5,9 & \begin{tabular}{|l|l}
5,9 \\
\end{tabular} & 4,7 & 3,5 & $5 \mid 4,2$ & 3,6 & $|3,2| 3,4 \mid$ & 4,1 & $|3,6|$ & 3,8 \\
\hline
\end{tabular}

Im ganzen zeigt es sich, daß die Geisteskrankheit bei ungefähr einem Drittel aller Fälle eine Dauer von weniger als 3 Monaten hatte; bei einem anderen Drittel dauerte sie schon länger als $1 \mathrm{Jahr}$; bei $1 / 4$ bis $1 / 5$ Teil 3--12 Monate, bei ungefähr $1 / 12$ währte sie schon seit der ersten Jugend. Die Verhältnisse waren ziemlich gleich bei beiden Geschlechtern; nur waren etwas mehr Männer geisteskrank seit der ersten Jugend.

Vergleicht man nun die verschiedenen Quinquennia miteinander, so würde hervorgehen, daß das Verhältnis derjenigen, die von $0-3$ oder von 3-12 Monaten, also innerhalb des ersten Jahres ihrer Krankheit aufgenommen wurden, kleiner geworden ist, $d . h$. daß man länger als früher mit der Aufnahme in eine Anstalt gezögert hat.

Um diese merkwürdige Tatsache erklären zu können, die man doch mit Rücksicht auf die vielen Verbesserungen in der Anstaltspflege am allerwenigsten erwarten würde, könnte man darauf hinweisen, daß gegenwärtig die Anamnese mit größerer Genauigkeit festgestellt wird als früher. Dies halte ich jedoch für eine schwache Argumentation, denn man denkt immer allzuleicht, daß man selbst eine Arbeit besser macht als ein anderer, der dasselbe verrichtet. Es gibt aber noch ein anderes Argument, das meines Erachtens viel wichtiger ist und erklärt, wie diese Veränderung nur scheinbar ist. Bei den Aufgenommenen sind nämlich auch diejenigen mitberechnet, welche nur aus einer Anstalt in eine andere übergingen, und vermutlich waren diese wohl meistens länger als ein Jahr geisteskrank. Wenn dies mit in Rechnung gezogen wird, wird der ohnehin geringe Unterschied keinen überwiegenden Einfluß mehr haben und bleibt die Dauer der Geisteskrankheit vor der Aufnahme in eine Anstalt wohl ziemlich dieselbe als früher. Auch hier ist also kein Faktor zu finden, der auf den Verlauf der Geisteskrankheit einen bedeutenden positiven oder negativen Einfluß gehabt hat. 
Art der Psychosen. Endlich müssen wir noch die Art der Psychosen bei den aufgenommenen Kranken feststellen, und kommen wir dadurch in eigentümliche Schwierigkeiten, bei denen wir erst einen Augenblick verweilen wollen. Die Angaben sind immer von der offiziellen Statistik mit ihrer eigenenNomenklatur abhängig; dieselbe hat noch obendrein in den letzten Jahren mehrfache Veränderungen erfahren. Die verschiedenen Krankheitsfälle mußten also so gut wie möglich in die eine oder die andere Rubrik untergebracht werden, und das war nicht immer leicht.

Dazu kommt noch eine andere Schwierigkeit, die nicht gering ist, und die darin besteht, daß das Zahlenmaterial für die Statistik von verschiedenen Beobachtern geliefert wurde, wobei jeder nach eigenem Gutdünken handelte. Aber diese Schwierigkeiten sind nun einmal nicht aus dem Wege zu räumen, ebensowenig als die Tatsache, daß diejenigen, welche von der einen Anstalt in die andere übergingen, nicht besonders erwähnt sind.

Tabelle 5. Psychosen bei den aufgenommenen Kranken.

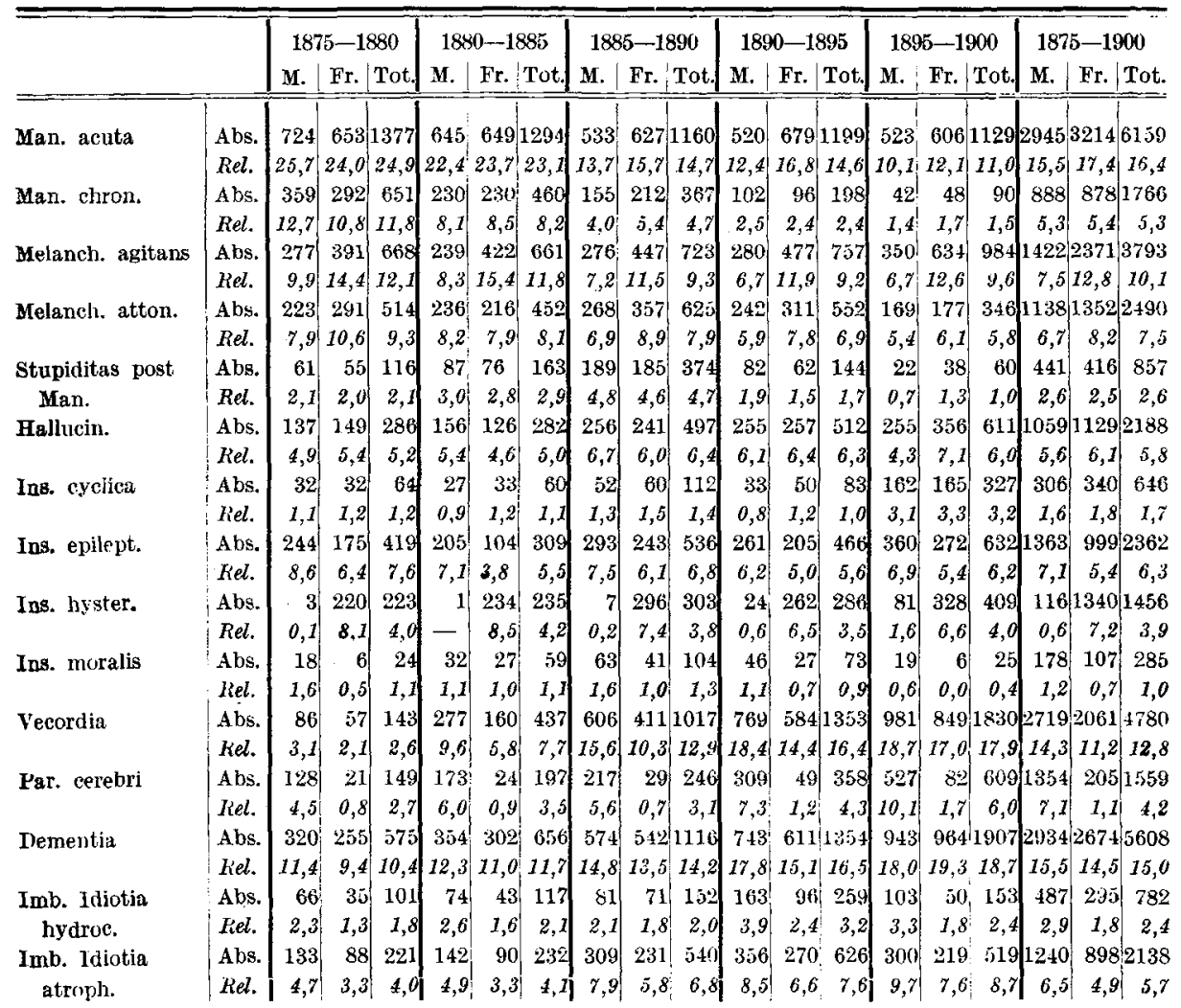


In dieser Tabelle wird eine Úbersicht gegeben der verschiedenen Krankheiten, die bei den aufgenommenen Kranken angegeben wurden. Es zeigt sich daraus, daß die meisten Patienten bei der Aufnahme an Mania acuta litten, eine große Anzahl an Dementia und Vecordia; weiter kommen Melancholia agitans und Melancholia attonita.

Von größter Wichtigkeit ist jetzt die Frage, ob bei den verschiedenen Psychosen eine Veränderung nachweisbar ist, d. h. ob eine relative $\mathrm{Zu}$ - oder Abnahme einiger Psychosen konstatiert werden kann. Es sind besonders vier Psychosen, die in dieser Hinsicht unsere Aufmerksamkeit in Anspruch nehmen, weil je zwei dieser eine Veränderung in positivem oder negativem Sinn zeigen. Bei Dementia und bei Paranoia ist eine bedeutende Zunahme wahrzunehmen, während die Zahl der Erkrankungen an Mania und Melancholia stark im Abnehmen begriffen ist. Man würde geneigt sein, dies Ergebnis als ein sehr wenig befriedigendes zu betrachten, zumal es bekannt ist, daß die sogenannten affektiven Psychosen im allgemeinen eine ziemlich günstige Prognose darbieten, während Dementia und Vecordia, wenn auch nicht eine absolut ungünstige, doch eine sehr zweifelhafte Prognose geben.

Jedoch darf man nicht außer acht lassen, daß es durchaus nicht unwahrscheinlich ist, daß man es nicht mit einerVeränderung in der Krankheit selber, sondern nur mit einer Veränderung im Namen zu tun hat. Es ist sehr gut möglich, daß die Krankheitsfälle, die früher mit dem Namen Mania oder Melancholia bezeichnet wurden, jetzt als Dementia gebucht werden, weil sie in die moderne Rubrik der Dementia praecox gebracht werden. Auch die Vecordia liefert von dieser Veränderung einen treffenden Beweis. Durch den Einfluß der Kraepelinschen Schule ist zu erwarten, daß der Begriff Vecordia wieder geringer wird und statt dessen die Gruppe Dementia, nämlich Dementia paranoides, tritt.

Bei den anderen Psychosen ist das Verhältnis in diesen 25 Jahren fast dasselbe geblieben, wenigstens ist keine regelmäßige Veränderung wahrzunehmen. die für den Verlauf der Geisteskrankheit von Bedeutung sein könnte.

Fassen wir zum Schluß alles zusammen, so zeigt sich, daß zwar die Zahl der Aufnahmen bedeutend gestiegen ist, daß jedoch mit Bezug auf das Alter der Kranken, auf die Rückkehr, auf die Dauer sowie auf die Art der Psychosen keine merkliche bedeutende Veränderung zu finden ist. Wir können also nicht feststellen, daß bei den aufgenommenen Kranken irgendein Faktor einen bedeutend günstigen oder ungünstigen Einfluß auf den Verlauf der Geisteskrankheit ausgeübt hat.

\section{Die Heilungen.}

Es versteht sich, daß bei der großen Zunahme der Anstaltsbevölkerung auch die Zahl der geheilten Kranken bedeutend größer geworden 
ist. Wenn man aber die Verhältnisse auf 100 Aufnahmen berechnet und miteinander vergleicht, da sind die Ziffern nicht so günstig, was sich ergibt aus der folgenden Tabelle, wo die durchschnittlichen Zahlen für jedes Dezennium in den Jahren 1850-1900 angegeben sind.

Tabelle 6. Ubersicht der Durchschnittszahlen der Geheilten.

\begin{tabular}{c|c|c|c|c|c|c}
\hline & \multicolumn{3}{|c|}{ Absolute Zahlen } & \multicolumn{3}{c}{$\begin{array}{c}\text { Relative Zahlen, } \\
\text { berechnet auf 100 Aufnahmen }\end{array}$} \\
& Männer & Frauen & Insgesant & Männer & Frauen & Insgesamt \\
\hline $1850-1860$ & 106 & 125 & 231 & 35,2 & 41,0 & 38,1 \\
$1860-1870$ & 158 & 180 & $\mathbf{3 3 8}$ & 36,5 & 41,2 & 38,9 \\
$1870-1880$ & 187 & 212 & 399 & 35,8 & 41,4 & 38,6 \\
$1880-1890$ & 185 & 217 & 402 & 30,7 & 36,5 & 33,5 \\
$1890-1900$ & 249 & 292 & 541 & 31,5 & 38,6 & 35,0
\end{tabular}

Eine deutliche Abnahme der Geheilten wurde besonders im vierten Dezennium, in den Jahren 1880-1890, beobachtet, was wahrscheinlich zusammenhing mit der Finführung des neuen Gesetzes für die Irrenpflege im Jahre 1884. Dadurch wurde doch eine große Anzahl chronischer Kranken den Anstalten zugeführt, wodurch das Verhältnis der Geheilten sehr herabgedrückt wurde.

Dieser Umstand macht es jedoch nicht verständlich, daß auch im letzten Dezennium das Verhältnis so niedrig geblieben ist, sogar niedriger als im ersten, ebenso bei dem männlichen als bei dem weiblichen Geschlecht. Was mag hiervon die Ursache sein?

Zur Beantwortung dieser Frage ist es wieder notwendig, einige Besonderheiten bei den Geheilten etwas näher ins Auge zu fassen. Das Zahlenmaterial dafür entnehmen wir den offiziellen Jahresberichten, welche vermelden, da $\beta$ in den Jahren $1875-1900$ als geheilt aus den Anstalten entlassen sind 11442 Kranke, von welchen 5282 Männer und 6160 Frauen, und wenn man die Verhältnisse berechnet auf 100

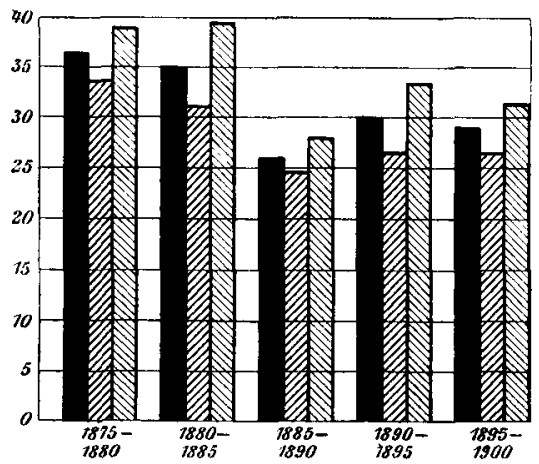

F'ig. 2. Verhältnisse der geheilten Kranken in jedem Quinquennium.
Kranke, die in derselben Zeit aufgenommen sind, $30,6 \%$, wobei das Verhältnis beiden Männern $(27,8 \%)$ bedeutend kleiner ist als bei den Frauen $(33,4 \%)$.

Wenn man jetzt die Verhältniszahlen für jedes Quinquennium berechnet und diese miteinander vergleicht, so ergeben sich ziemlich bedeutende Differenzen, wovon man eine deutliche Úbersicht be. kommt in Fig. 2.

Es ergibt sich, daß im ersten 
Quinquennium verhältnismäßig die meisten Kranken geheilt sind, danach wurde ihre Zahl weniger und in den beiden letzten Perioden ist sie wieder etwas gestiegen, doch immer bleibt sie noch kleiner als im Anfang; der Einfluß des neuen Irrengesetzes von 1884 ist hier sehr deutlich bemerklich im dritten Quinquennium.

Von den beiden Geschlechtern ist immer das Verhältnis bei den geheilten Frauen etwas größer als bei den Männern; ein Regelmaß kann man jedoch nicht beobachten, denn in der zweiten Periode war die Dif ferenz $8,8 \%$ und in der dritten nur $2,4 \%$.

Alter. Die Frage ist jetzt: wie steht es denn mit dem Alter der Kranken, die in dieser Zeit aus den Anstalten geheilt entlassen sind?

Ich habe die diesbezüglichen Zahlen der offiziellen Statistik in eine Tabelle zusammengebracht und eine Utbersicht dargestellt der Kranken, die in demselben Alter genesen sind; sogleich habe ich dabei die prozentweisen Verhältnisse beigegeben.

Tabelle 7. Alter der gebeilten Kranken.

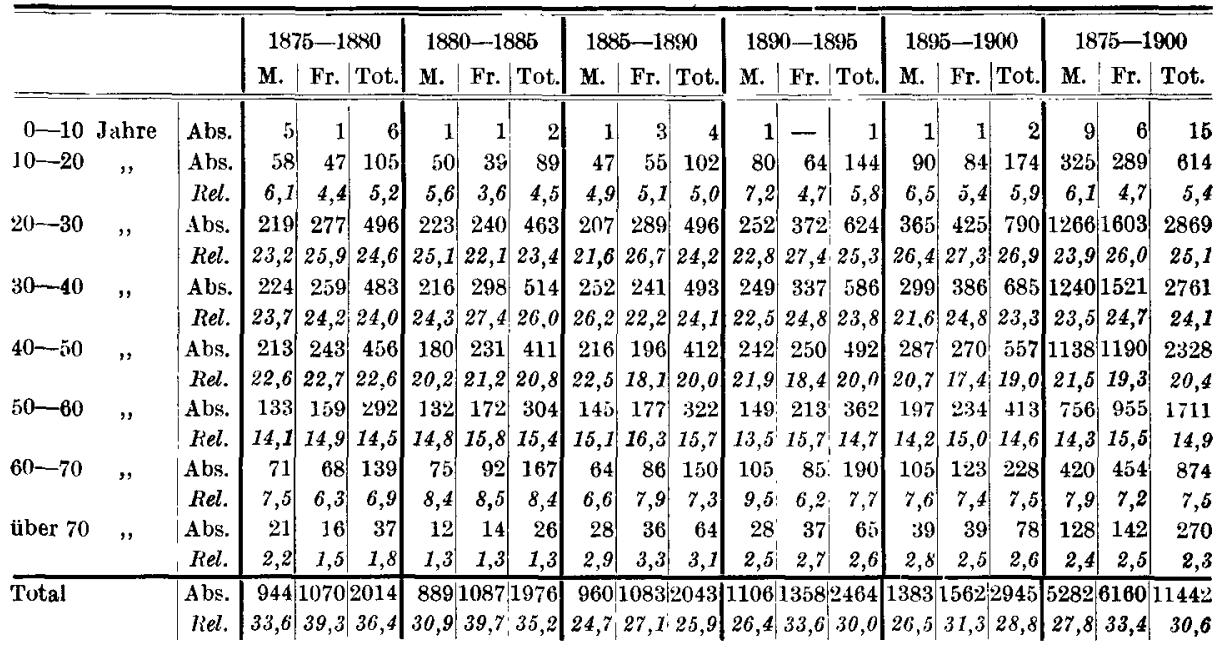

Was zunächst die ganze Periode betrifft, ist beinahe die Hälfte genesen in dem Alter von 20-40 Jahren; in dieser Periode ist also die Möglichkeit einer Heilung am größten. Die Zahl der Geheilten unter 20 Jahren ist verhältnismäßig gering und beträgt nur 5,4\%; nach 40 Jahren nimmt die Genesung regelmäßig ab, und im Alter höher als 70 Jahre kam sie nur bei 2,3\% vor. Eine deutliche Utbersicht dieser Zahlen sieht man in Fig. 3, wo auch die Verhältnisse für Männer und Frauen apart angegeben sind. 
Hat nun in dieser Hinsicht eine Änderung stattgefunden? Gibt es jetzt mehr Kranke einer bestimmten Altersstufe, die geheilt entlassen

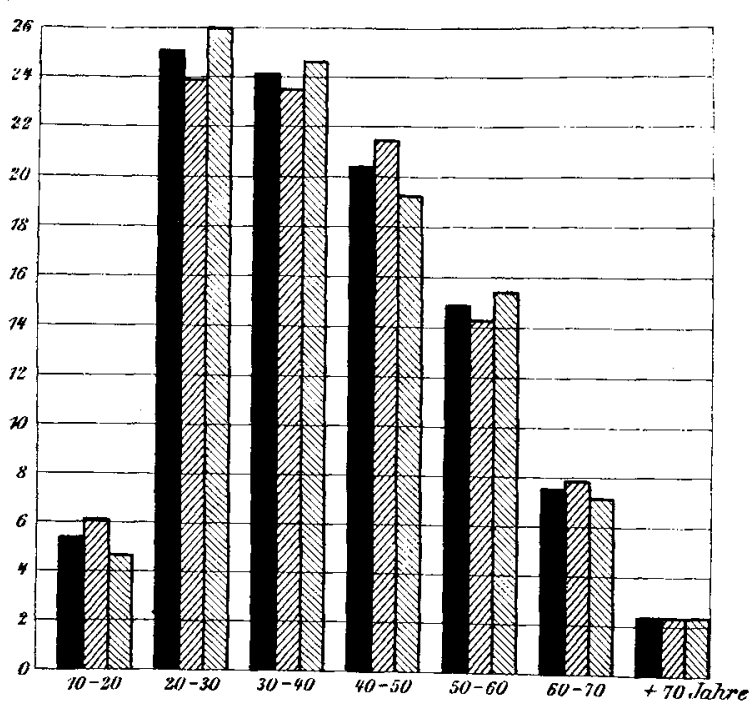

Fig. 3. Verteilung der geheilten Kranken auf die verschiedenen Altersstufen.

(11 442 Patienten; 5282 Männer, 6160 Frauen.) wurden, als früher? Wenn man die darauf bezüglichen Zahlen miteinander vergleicht, dann stellt sich heraus, daß die Zahl an Geheilten unter 30 und über 60 Jahre verhältnismäßig um ein wenig gestiegen ist,während diese Zahl zwischen 40 und 50 Jahren ein wenig sich verminderte. Diese Unterschiede sind jedoch so gering, daß sie keine große Bedeutung haben können. Auch

im Verhältnis zwischen beiden Geschlechtern kam keine wesentliche Veränderung zustande. Wir kommen also zu dem Ergebnis, daß im Alter der Geheilten keine bedeutende Veränderung zustande gekommen ist und die Zahlen ziemlich dieselben geblieben sind.

Dauer. Ist vielleicht infolge der viel verbesserten Anstaltspflege die Dauer derselben bei den Geheilten kürzer geworden?

Um eine Antwort auf diese Frage zu bekommen, habe ich aus den obengenannten Jahresberichten auch zusammengestellt, wie lange die geheilt Entlassenen in der Anstalt gepflegt wurden. Ich habe diese Zahlen berechnet für die gesamte Periode und für jedes Quinquennium apart und auch die Verhältnisse angegeben für 100 Kranke (Tabelle 8).

Im ganzen stellt sich heraus, daß etwas weniger als die Hälfte der Geheilten kürzer als 6 Monate und nicht ganz drei Viertel derselben kürzer als 12 Monate in der Anstalt gepflegt wurden. Nach einer Pflege von 4 Jahren wurden noch $4,3 \%$ als geheilt entlassen, und nach 10 Jahren $0,9 \%(0,8 \%$ der Männer, $1 \%$ der Frauen). In den ersten 3 Monaten wurden mehr Männer als Frauen geheilt; im zweiten Tremester ist das Verhältnis ein umgekehrtes. Im beiderseitigen Verhältnis zeigen sich in dem ganzen Zeitraum einige Schwankungen, doch regelmäßige Veränderungen sind nicht wahrzunehmen.

Im ganzen ist also keine Änderung in der Dauer der Anstaltspflege 
zu bemerken und auf keinen Fall ist die Dauer derselben kürzer geworden. Man sollte meinen, daß bei der viel verbesserten Pflege die Kranken jetzt früher geheilt seien, aber aus unseren Zahlen könnte man dies nicht beweisen. Und daß sogar nach mehr als vierjähriger Pflege noch so viele Kranke als geheilt entlassen wurden, beweist wohl, daß der Begriff des Geheiltseins nicht allzu wörtlich aufgefaßt wird.

Tabelle 8. Pflegezeit der geheilten Kranken.

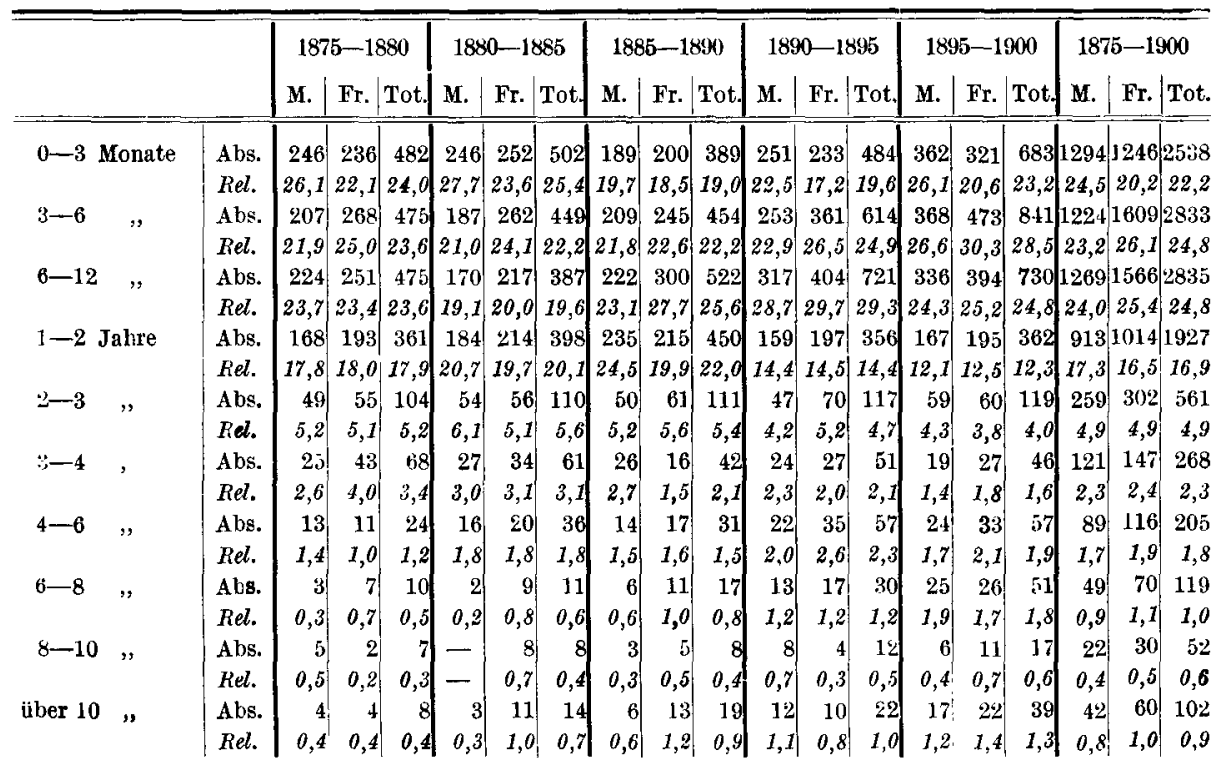

Art der Psychosen. Endlich kommt noch die Frage, welche Psychosen denn bei den Geheilten vorgekommen sind.

In bezug auf diese Frage habe ich die folgende Tabelle zusammengestellt, die eine Übersicht gibt der verschiedenen Psychosen, die in dem ganzen Zeitraum und in jedem Quinquennium apart bei den Geheilten beobachtet wurden; zur besseren Vergleichung habe ich auch überall die Verhältnisse angegeben auf 100 Kranke, die in derselben Zeit genesen sind (Tabelle 9).

Es stellt sich aus dieser Tabelle heraus, daß angeblich die meisten Kranken, die als geheilt entlassen wurden, gelitten haben an Mania acuta $(33,6 \%)$ und dann folgte Melancholia agitans; weiter verdienen noch besondere Beachtung Melancholia attonita mit $9.6 \%$ und Vecordia mit 9,9\%. Daß man aber den Ausdruck ,geheilt sein" nicht buchstäblich nehmen muß, geht wohl daraus hervor, daß bei Psychosen, wie Insania moralis und Paralysis cerebri mehrere Fälle als geheilt angegeben sind, sogar auch Fälle von Imbecillitas und Idiotia. 
Tabelle 9. Art der Psychosen bei den Geheilten.

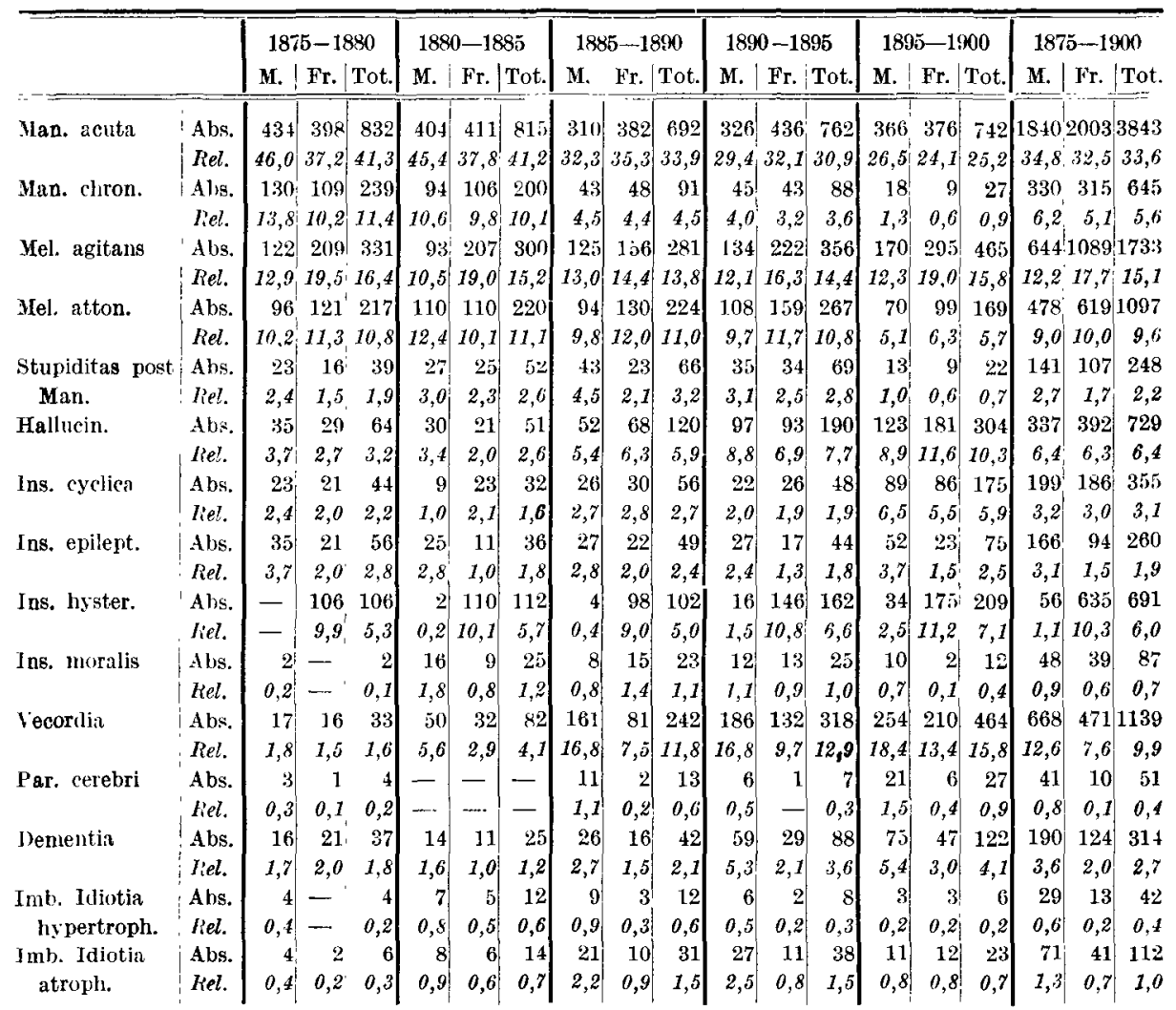

Ist nun irgendeine Veränderung in dem Zahlenverhältnis der Geheilten während der genannten Zeit entstanden? Bei mehreren Psychosen kann deutlich eine entschiedene Abnahme beobachtet werden; ich weise dazu auf Mania acuta, Mania chronica, Melancholia attonita. Bei anderen dagegen kann eine bedeutende Zunahme der Verhältnisse konstatiert werden, z. B. bei Hallucinationes, Insania cyclica, Vecordia und Dementia.

Zur richtigen Beurteilung muß hierbei jedoch in Betracht genommen werden, daß in den letzten Jahren wichtige Veränderungen in der Diagnose zustande gekommen sind. Statt der früheren Diagnose ,Hallucinationes" spricht man jetzt von Amentia, und vermutlich ist die Zahl der Geheilten bei Mania dadurch so sehr vermindert, daß jetzt viele Fälle bei der Amentia oder Vecordia untergegebracht sind. Ein bestimmter Schluß, daß im Zahlenverhältnis der Geheilten eine deutliche Veränderung stattgefunden hat mit Bezug auf die Art der Psychose, kann nicht angenommen werden. 
Zum Schluß gibt es noch eine Tatsache, auf welche ich ganz besonders die Aufmerksamkeit richten möchte. Aus dem letzten Berichte der staatlichen Inspektoren über die Jahre 1900, 1901 und 1902, Seite 202, ergibt sich, daß in dieser Zeit 35,3\% als geheilt entlassen wurden. Das Verhältnis aller Kranken, die seit 1844 in die Anstalt aufgenommen wurden und diese wieder als geheilt verlassen haben (ihre Anzahl beträgt 18021 ), ist 35,7\%. Im Laufe von fast 60 Jahren ist also keine nennenswerte Verbesserung in den Heilungsprozenten wahrzunehmen, und durchschnittlich bleibt die Zahl der Geheilten noch immer dieselbe.

\section{Die Todesfälle.}

Betrachten wir jetzt die Zahl der Todesfälle in den Anstalten, so versteht es sich von selbst, daß diese mit der größeren Bevölkerung sich auch bedeutend vermehrt hat. Wenn man aber die Verhältnisse vergleicht auf 100 Anstaltsinsassen, so sind die Ziffern etwas günstiger, wie sich zeigt aus der folgenden Tabelle, wo die Durchschnittszahlen in jedem Dezennium der Jahre 1850-1900 gegeben werden.

Tabelle 10. Úbersicht der durchschnittlichen Todesfälle.

\begin{tabular}{|c|c|c|c|c|c|c|}
\hline & \multicolumn{3}{|c|}{ Absolute Zablen } & \multicolumn{3}{|c|}{$\begin{array}{c}\text { Relative Zahlen, } \\
\text { berechnet auf } 100 \mathrm{Kranke}\end{array}$} \\
\hline & Männer & Frauen & Insgesant & Männer & Frauen & Insgesamt \\
\hline $1850-1860$ & 118 & 93 & 211 & 10,6 & 7,8 & 9,2 \\
\hline $1860-1870$ & 162 & 146 & 307 & 9,4 & 8,0 & 8,7 \\
\hline $1870-1880$ & 224 & 186 & 410 & 9,6 & 7,6 & 8,6 \\
\hline $1880-1890$ & 263 & 222 & 485 & 8,8 & 7,1 & 7,9 \\
\hline $1890-1900$ & 322 & 281 & 603 & 7,6 & 6,7 & 7,2 \\
\hline
\end{tabular}

Es zeigt sich also, daß die Mortalität regelmäßig kleiner geworden ist nicht nur im ganzen, sondern auch bei dem männlichen und weiblichen Geschlecht apart. Diese Abnahme, die allmählich zustande gekommen ist, war auch à priori sehr wahrscheinlich mit Bezug auf die verbesserte Anstaltspflege. Sehr auffallend ist die Tatsache, daß die Mortalität bei den Männern viel stärker abgenommen hat als bei den Fraven, doch bleibt die Mortalität bei den Frauen am geringsten.

Die Frage ist jetzt, ob es mit Bezug auf die Todesfälle in den Anstalten noch einige Besonderheiten gibt, die von allgemeiner Bedeutung sind. Es ist nötig, dazu die Zahlen aus den offiziellen Jahresberichten etwas näher zu betrachten: diese vermelden, daß in den Jahren 1875 bie 1900 verstorben sind 13056 Kranke, wovon 7076 Männer und 5977 Frauen, oder wenn man das Verhältnis berechnet auf 100 Aufnahmen, 34,9\%, wovon 37,3\% für die Männer und 32,3\% für die Frauen. 


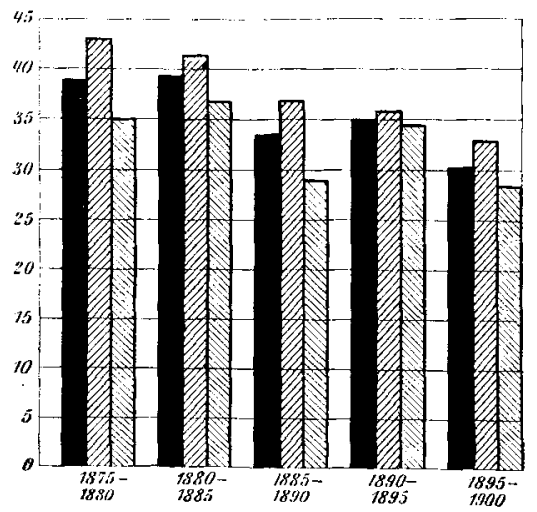

Tig. 4. Verhältnisse der verstorbenen Kranken in jedem Quinquennium.

Wenn man die durchschnittlichen Verhältnisse in jedem Quinquennium miteinander vergleicht, so ergibt sich eine ziemlich bedeutende Abnahme der Mortalität von 39,3 bis $30,7 \%$, welche Abnahme aber nicht ganz regelmäßig war. Bei den Männern war die Mortalität immer etwas höher als bei den Frauen; in der ersten Periode war der Unterschied $8,3 \%$, in der letzten jedoch nur $4,4 \%$

Alter. Zuerst beantworten wir jetzt die Frage, wie es steht mit dem Alter der Verstorbenen. In der folgenden Tabelle habe ich eine Utbersicht zusammengestellt, wie viele Kranke in jedem Lebensalter verstorben sind. Diese Zahlen wurden berechnet für die ganze Zeit und für jedes Quinquennium, auch wurden die Verhältnisse angegeben auf 100 Todesfälle.

Tabelle 11. Alter der Verstorbenen.

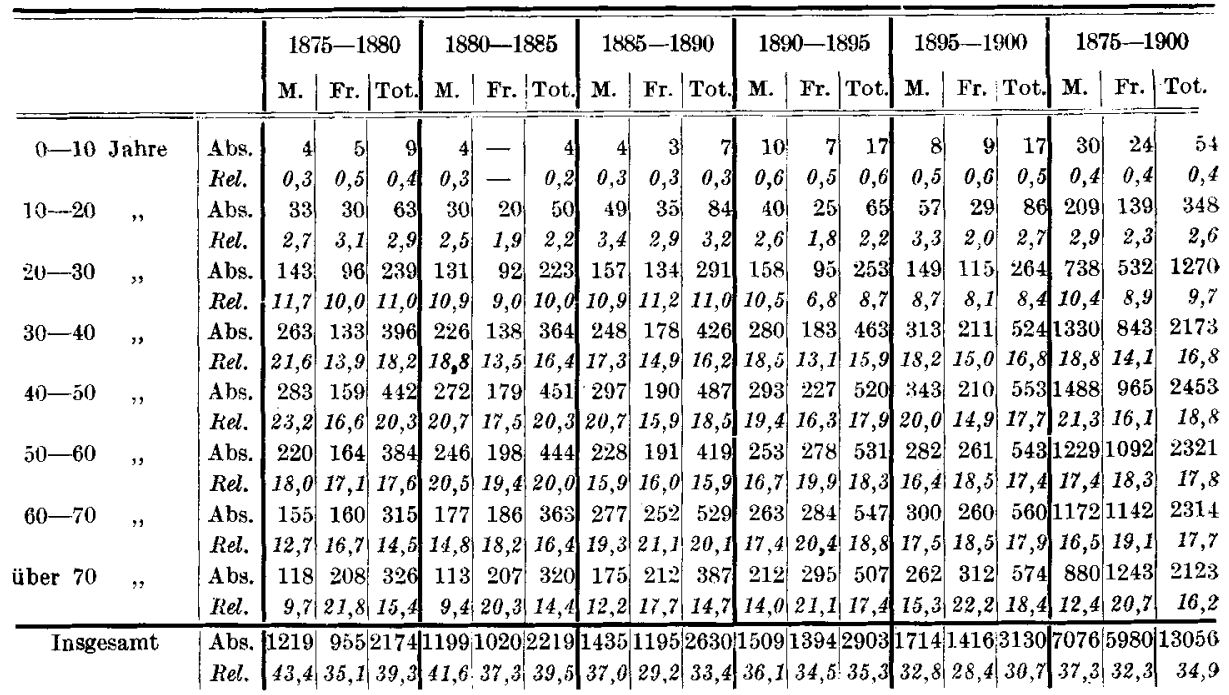

Es ergibt sich aus dieser Tabelle, daß im ganzen die Mortalität am größten war zwischen 40 und 50 Jahren, aber sie war in den beiden folgenden Dezennien nur wenig geringer. Bei den Männern war sie 
am größten zwischen 40 und 50 Jahren, bei den Frauen war sie jedoch erst im späteren Lebensalter am größten; 20,7\% der verstorbenen Frauen hatte ein Alter höher als 70 Jahre.

Wenn man die verschiedenen Quinquennia miteinander vergleicht, so zeigt sich, daß die Mortalität bei den Kranken von 20-50 Jahren, also in der besten Kraft des Lebens, sich etwas vermindert hat, während sie im Alter von 60 Jahren und darüber zugenommen hat. Dies

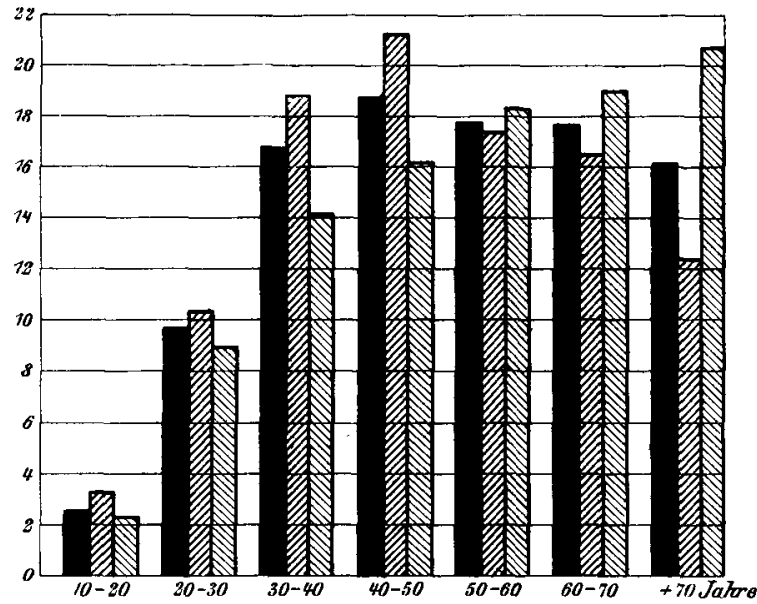

Fig. 5. Verteilung der verstorbenen Kranken auf die ver. sehiedenen Altersstufen.

(13056 Patienten; 7076 Mānner, 5980 Frauen.)

ist von großer Wichtigkeit und jedenfalls das Resultat der besseren hygienischen Zustände und veränderten Pflege in den Anstalten. Hinsichtlich der Geschlechter starben verhältnismäßig mehr Männer, die die 50 Jahre noch nicht erreicht hatten, und mehr Frauen, die dieses Alter überschritten hatten. Im Alter von 70 Jahren und darüber ist dieses Verhältnis bei den Frauen sogar mehr als $8 \%$ höher.

Es geht daraus hervor, daß der Verlauf der Psychosen bei den Männern im allgemeinen sich ungünstiger gestaltet als bei den Frauen.

Dauer. Wie steht es denn mit der Dauer der Anstaltspflege bei den Verstorbenen? Ist diese Zeit länger oder kürzer als früher?

Eine Utbersicht der Zeit, während welcher die verstorbenen Kranken in der Anstalt gepflegt wurden, findet man in Tabelle 12.

Es zeigt sich aus dieser Tabelle, daß ungefähr zwei Fünftel der ganzen Anzahl der Verstorbenen schon innerhalb des ersten Jahres ihrer Aufnahme gestorben sind, während 17,2\%, also nicht ganz ein Fünftel, länger als 10 Jahre gepflegt wurden, ehe der Tod sie dahinraffte. Auch hier finden wir einen bedeutenden Unterschied bei beiden Geschlechtern: in den ersten 3 Jahren der Pflege starben verhältnis- 
mäßig mehr Männer, später mehr Frauen; bei denen, die nach einer Pflege von mehr als 10 Jahren starben, war das Verhältnis der Frauen reichlich 4\% mehr als das der Männer. Der Verlauf der Psychosen war also auch hier bei den Männern ungünstiger als bei den Fraven.

Tabelle 12. Pflegezeit der Verstorbenen.

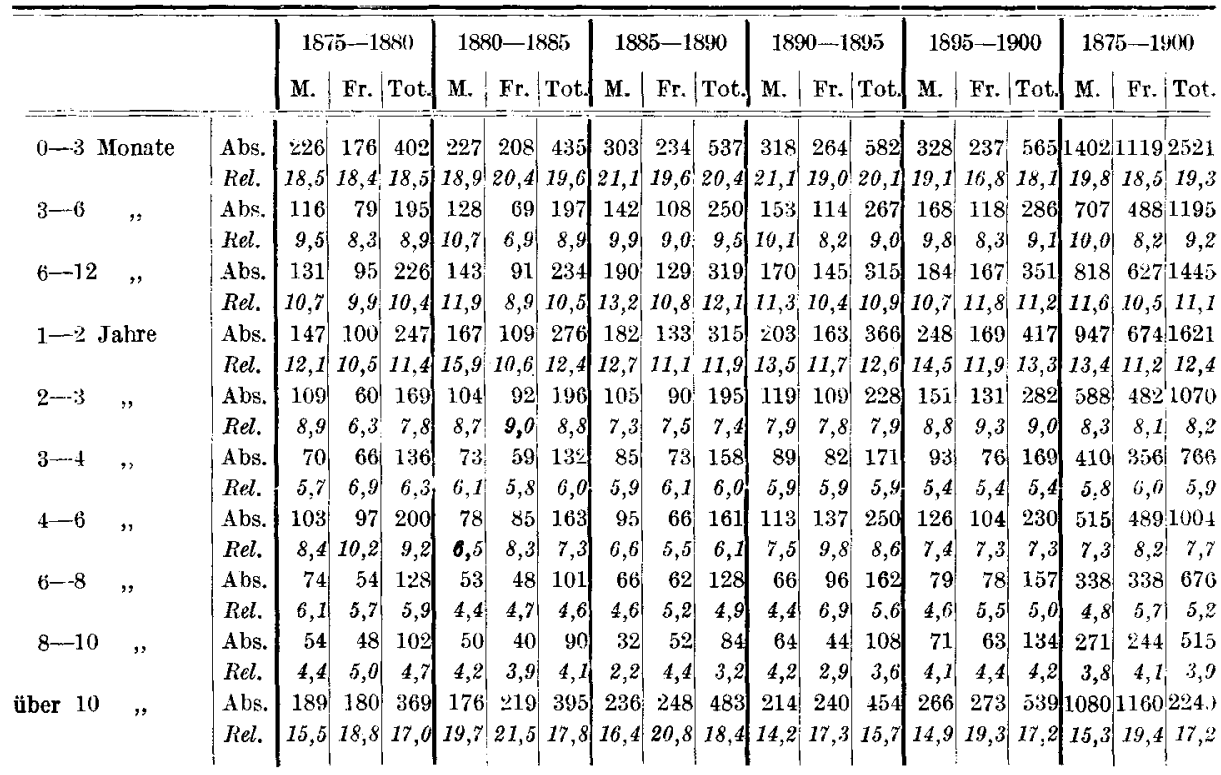

Von großer Wichtigkeit ist jetzt die Frage, ob in diesem Zeitraum eine Änderung in der Dauer der Pflegezeit bei den Verstorbenen stattgefunden hat. Es fragt sich, ob eine Neigung zur Verlängerung oder zur Verkürzung der Pflege könnte beobachtet werden. Wird z. B. die Anzahl derjenigen größer, welche bei ihrem Tode schon längere Zeit in der Anstalt gepflegt wurden? Oder ist eine deutliche Veränderung zu entdecken in der Zahl derer, die schon nach einigen Monaten in der Anstalt gestorben sind?

Es hat sich keines von beiden gezeigt. Die Zahlen sind mit einigen kleinen Schwankungen größtenteils dieselben geblieben. A priori würde man wohl erwarten können, daß die Behandlung insofern einen günstigen Eindruck ausgeübt hätte, daß die Zahl derjenigen, welche innerhalb weniger Monate gestorben sind, vermindert wäre; aus unseren Zahlen ist das jedoch nicht zu beweisen. Während also die Zahl der Todesfälle nur verhältnismäßig wenig vermindert ist, zeigt sich, daß die Dauer der Pflege im ganzen nicht bedeutend anders geworden ist. Einen deutlichen Einfluß auf die Zeit der Pflege hat die modarne Behandlung also nicht ausgeübt. 
Art der Psychosen. Zum Schluß folgen noch einige Besonderheiten über die Art der Psychosen, welche bei den Verstorbenen beobachtet wurden. Die folgende Tabelle zeigt, welche Psychosen in dem ganzen Zeitraum und in jedem Quinquennium bei den Verstorbenen vorgekommen sind; auch wird zur besseren Vergleichung das Verhältnis jeder Psychose angegeben mit Bezug auf 100 Todesfälle.

Tabelle 13. Art der Psychosen bei den Verstorkenen.

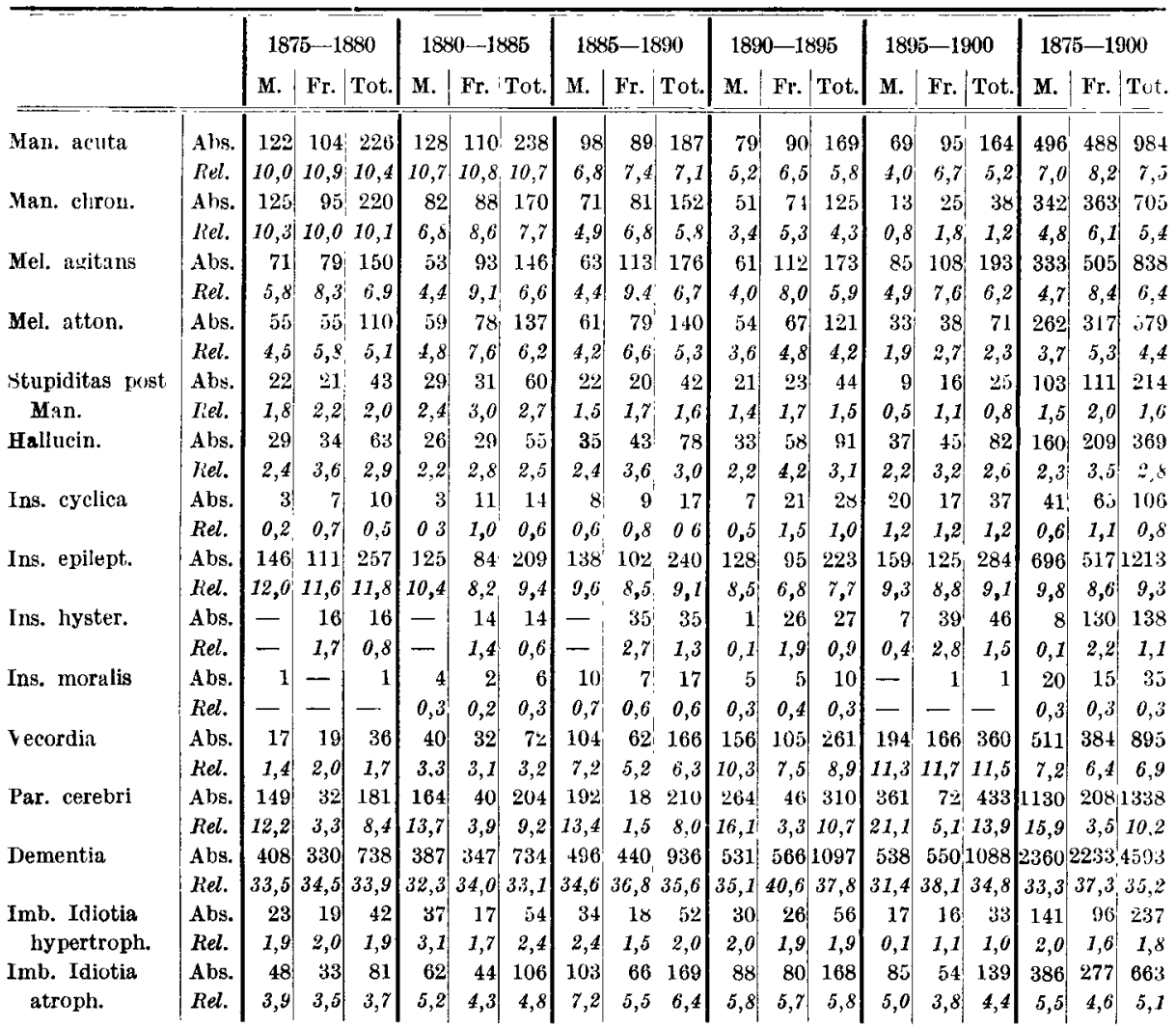

Die größte Mortalität wurde beobachtet bei der Dementia, dann folgen Insania epileptica, Paralysis Cerebri, Mania acuta usw. Die Verhältnisse zeigen einige Differenzen nach dem Geschlechte bei Paralysis cerebri und bei Dementia.

Hat sich nun die Ziffer der Mortalität in der Zeit von 1875-1900 irgendwo bei den Psychosen bedeutend geändert? Es zeigt sich, daß bei mehreren Psychosen das Verhältnis kleiner geworden ist; ich nenne besonders dazu Mania acuta und Mania chronica, während bei Vecordia 
und Paralysis cerebri die Mortalität sich vergrößert hat. Wahrscheinlich war hier aber zum Teile auch eine Änderung in der Benennung der Krankheit von Einfluß, und wird in vielen Fällen, die früher als Mania acuta oder chronica bezeichnet wurden, jetzt die Diagnose Paranoia (Dementia paranoides) gemacht. Auch übt natürlich das, was über die Nomenklatur bei den Aufnahmen gesagt wurde, bedeutenden Einfluß aus.

Wir kommen also zu dem Ergebnis, daß bei den verstorbenen Kranken die Art der Psychose nicht gestattet, einen Schluß zu ziehen auf einen anderen Verlauf der Psychosen; man kann nicht beweisen, daß die eine oder andere Psychose jetzt besser oder schlechter verläuft als früher.

\section{SchluB.}

Wenn wir zum Schluß alles noch einmal kurz zusammenfassen, so zeigt sich das folgende:

1. Die Zahl der in die Irrenanstalten aufgenommenen Kranken nimmt fortwährend zu.

2. Die Zahl der als geheilt aus diesen Anstalten entlassenen Kranken wird geringer.

3. Die Zahl der Kranken, die in den Anstalten verstorben sind, wird ebenso geringer.

4. Die Bevölkerung der Irrenanstalten muß also beständig zunehmen.

Es zeigt sich aus den offiziellen Berichten, daß die Zahl der Geisteskranken in den niederländischen Irrenanstalten in der letzten Hälfte des vorigen Jahrhunderts sich absolut und relativ sehr bedeutend vermehrt hat. Die folgende Tabelle gibt eine Übersicht der Kranken, die durchschnittlich in jedem Dezennium gepflegt wurden; auch wird angegeben das Verhältnis auf 10000 Einwohner.

Tabelle 14. Ubersicht der Geisteskranken in den Anstalten.

\begin{tabular}{c|r|r|r|r|r|r}
\hline & \multicolumn{3}{|c|}{ Gesamtzahl im Durchschnitt } & \multicolumn{3}{|c|}{$\begin{array}{c}\text { Verhältniszahl auf 10000 Einwohner } \\
\text { im Durchschnitt }\end{array}$} \\
& Männer & Frauen & Insgesamt & Männer & Frauen & Insgesamt \\
\hline $1850-1860$ & 797 & 864 & 1661 & 5,0 & 5,3 & 5,2 \\
$1860-1870$ & 1277 & 1389 & 2665 & 7,4 & 7,9 & 7,7 \\
$1870-1880$ & 1800 & 1921 & 3721 & 9,7 & 10,1 & 9,9 \\
$1880-1890$ & 2404 & 2504 & 4908 & 11,4 & 11,6 & 11,5 \\
$1890-1900$ & 3440 & 3459 & 6899 & 14,5 & 14,3 & 14,4
\end{tabular}

Die Vermehrung der Kranken ist also sehr groß und gestiegen von $1661 \mathrm{im}$ ersten bis $6899 \mathrm{im}$ letzten Dezennium, also mehr als viermal größer geworden. Die Verhältniszahl auf 10000 Einwohner ist gestiegen von 5,2 auf 14,4 , und diese Vermehrung war ziemlich regelmäßig. Jedes Jahr nimmt die Anstaltsbevölkerung mit $200 \mathrm{zu}$, oder 
um 0,3 auf jede 10000 Einwohner, eine Zunahme, die ernstliche Besorgnis für die $\mathrm{Zu}$ kunft erwecken muß.

Wenn man die Zahl der Anstaltsbevölkerung nimmt über die Jahre 1875-1900 und die Durchschnittswerte für je $\mathbf{5}$ Jahre miteinander vergleicht, so ergibt sich eben dieselbe Zunahme. In dieser Zeit fand eine bedeutende Vermehrung der Zahl derer statt, die im ersten Quinquennium gepflegt wurden. Das Verhältnis ist gestiegen von 10,2 auf 15,0 auf 10000 Einwohner. Im allgemeinen hat die Zahl der Männer etwas mehr zugenommen als die der Frauen.

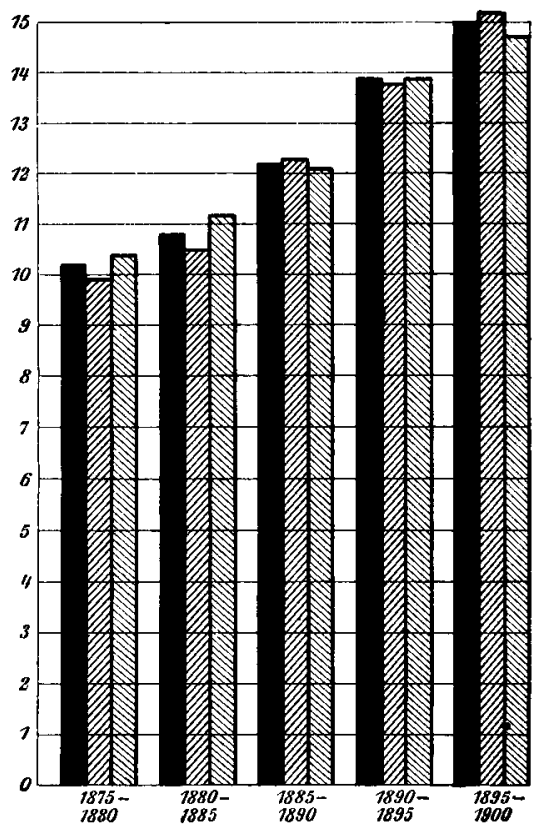

Fig. 6. Zahl der auf 10000 Einwohner in Anstalten verpflegten Geisteskranken.

Herkunft. Was die Herkunft der Kranken betrifft, ergibt sich, daß sie nicht regelmäßig über das ganze Land verteilt waren. Die folgende Tabelle gibt eine Utbersicht der Provinzen und der Geisteskranken, die zu jeder Provinz gehören; auch werden überall die Verhältniszahlen auf 10000 Einwohner angegeben (Tabelle 15).

Verhältnismäßig findet man die größte Zahl in der Provinz Utrecht; auf eine Bevölkerung von 215844 Einwohnern kamen 370 Geisteskranke, mit anderen Worten 17,0 auf 10000 Einwohner; dann folgt die Provinz Nordholland mit 16,6, Limburg mit 14,4, dann die anderen Provinzen. Am günstigsten gestaltet sich das Verhältnis für die Provinz Zeeland mit 6,6\% auf 10000 Einwohner, was vermutlich durch die isolierte Lage dieser Provinz zu erklären ist; vielleicht hat hierauf auch die Tatsache Einfluß, daß es in dieser Provinz keine einzige Anstalt gibt, und die Familien sich dagegen sträuben, die Patienten in entfernter gelegenen Anstalten behandeln zu lassen, solange dieses nicht dringend notwendig ist. Im ganzen zeigt sich im Zahlenverhältnis kein großer Unterschied zwischen den beiden Geschlechtern.

In allen Provinzen hat das Verhältnis in den letzten 25 Jahren regelmäßig zugenommen; am stärksten ist dies der Fall in Nordholland, doch auch in den übrigen Provinzen zeigt sich eine fortwährende $\mathrm{Zu}$ nahme, am wenigsten in den drei nördlichen Provinzen Friesland, 
Tabelle 15. Herkunft der Kranken nach den Provinzen.

\begin{tabular}{|c|c|c|c|c|c|c|c|c|c|c|c|c|c|c|c|c|c|c|c|}
\hline \multirow{3}{*}{ Ford-Brabant } & \multirow{3}{*}{$\begin{array}{l}\text { Tot. } \\
\text { :000 }\end{array}$} & \multicolumn{3}{|c|}{ 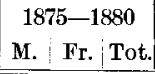 } & \multicolumn{2}{|c|}{$1880-1885$} & $\begin{array}{l}885 \\
\text { Tot. }\end{array}$ & \multicolumn{3}{|c|}{$1885-1890$} & \multicolumn{2}{|c|}{$1890-1895$} & $\begin{array}{l}895 \\
\text { Tot. }\end{array}$ & & \multicolumn{2}{|c|}{$1895-1900$} & $\begin{array}{l}187 \\
\text { M. }\end{array}$ & $\begin{array}{l}75-19 \\
\mid \text { Fr. }\end{array}$ & $\begin{array}{l}9(4) \\
\text { Tot. }\end{array}$ \\
\hline & & 239 & 260 & 500 & 252 & 301 & 553 & 351 & 343 & 694 & 401 & 335 & 735 & 462 & 380 & 843 & $3+1$ & 324 & 665 \\
\hline & & $10,2 \mid$ & 11,3 & 10,8 & 10,4 & 12,5 & 11,5 & 13,8 & 13,6 & 13,7 & 15,3 & 12,9 & 11,1 & 16,9 & 13,9 & 15,4 & 13,3 & 12,8 & 13,1 \\
\hline \multirow[t]{2}{*}{ Gelderland } & Tot. & 224 & 223 & 447 & 269 & 268 & 538 & 312 & 326 & 638 & 358 & 384 & 742 & 381 & 395 & 776 & 309 & 319 & 628 \\
\hline & $\%$ & 9,6 & 9,8 & 9,7 & 11,0 & 11,2 & 11,1 & 12,1 & 13,0 & 12,6 & 13,4 & 14,8 & 14,1 & 13,5 & 14,4 & 13,9 & 11,9 & 12,6 & 12,3 \\
\hline \multirow[t]{2}{*}{ Sud-Holland } & Tot. & 345 & 452 & 797 & 399 & 501 & 900 & 494 & 574 & 1068 & 589 & 679 & 1268 & 713 & 787 & 1500 & 508 & 599 & 1107 \\
\hline & $0 ! 000$ & 9,1 & 11,2 & 10,2 & 9,7 & 11,3 & 10,5 & 11,0 & 11,9 & 11,5 & 12,1 & 13,0 & 12,6 & 13,3 & 13,7 & $13,5 \mid$ & 11,0 & 12,2 & 11,7 \\
\hline \multirow[t]{2}{*}{ Nord-Holland } & T'ot. & 433 & 498 & 931 & 487 & 536 & 1023 & 627 & 670 & 1296 & 783 & 834 & 1617 & 937 & 991 & 1928 & 653 & 7061 & 1359 \\
\hline & $: 000$ & 13,6 & 14,7 & 14,2 & 13,7 & 14,2 & 14,0 & 16,1 & 16,1 & 16,1 & 18,4 & 18,4 & 18,4 & 20,4 & 20,2 & 20,3 & 16,4 & 16,7 & 16,6 \\
\hline \multirow[t]{2}{*}{ Zeeland } & Tot. & 49 & 48 & 97 & 58 & $5 t$ & 112 & 76 & 63 & 138 & 74 & 65 & 139 & 99 & 82 & $1 \times 1$ & 71 & 62 & 134 \\
\hline & 000 & 5,2 & 5,0 & 5,1 & 6,1 & $5, \overline{0}$ & 5,8 & 7,7 & 6,2 & 6,9 & 7,3 & 6,3 & 6,8 & 9,3 & $\tau, 6$ & 8,4 & 7,1 & 6,1 & 6,6 \\
\hline \multirow[t]{2}{*}{ Utrecht } & Tot. & 135 & 136 & 271 & $164 \mid$ & 169 & 333 & $\mid 172$ & 202 & 374 & $\mid 187$ & 226 & 413 & 222 & 238 & 460 & 176 & 194 & 370 \\
\hline & $0 ! 000$ & $14,5$. & 14,1 & 14,3 & 16,4 & 16,6 & 16,6 & 16,1 & 18,5 & 17,3 & 16,4 & 19,4 & 17,9 & 18,3 & 19,1 & 18,7 & 16,3 & 17,5 & 17,0 \\
\hline \multirow[t]{2}{*}{ Friesland } & Tot. & 105 & 110 & 215 & 115 & 130 & 245 & 144 & 141 & 285 & $154^{i}$ & 178 & 332 & 173 & 202 & 375 & 138 & 152 & 290 \\
\hline & ${ }_{0}^{0}, 000$ & 6,5 & 6,8 & 6,7 & 7,0 & 7,8 & 7,4 & 8,5 & 8,3 & 8,4 & 9,1 & 10,5 & 9,8 & 10,2 & 11,8 & 11,0 & 8,3 & 9,0 & 8,7 \\
\hline \multirow[t]{2}{*}{ Overyssel } & Tot. & 104 & 93 & 197 & 112 & 94 & 207 & 128 & 121 & 249 & 160 & 139 & 299 & 180 & 169 & 349 & 137 & 123 & 260 \\
\hline & 1000 & 7,5 & 7,0 & 7,2 & 7,7 & 6,9 & 7,3 & 8,5 & 8,4 & 8,5 & 10,3 & 9,2 & 9,8 & $\mid 10,9$ & 10,6 & 10,7 & 9,0 & 8,4 & 8,7 \\
\hline \multirow[t]{2}{*}{ Groningen } & Tot. & 76 & 82 & 158 & 87 & 95 & 182 & 110 & 137 & 247 & 119 & 156 & 275 & 161 & 182 & 344 & 111 & 130 & 241 \\
\hline & $0_{000}^{\prime}$ & 6,2 & 6,6 & 6,4 & 6,7 & 7,2 & 6,9 & 8,1 & 9,9 & 9,0 & 8,6 & 10,9 & 9,8 & 11,0 & 12,1 & 11,6 & $8, \mathbf{1}$ & 9,3 & 8,7 \\
\hline \multirow[t]{2}{*}{ Drenthe } & 'Tot. & 45 & 31 & 75 & 58 & 30 & 88 & 54 & 36 & 90 & 63 & 58 & 121 & 61 & 62 & 123 & 56 & 43 & 99 \\
\hline & $0: 000$ & 7,5 & 5,5 & 6,4 & 9.1 & 5,2 & 7,2 & 8,0 & 5,8 & 6,9 & 8,8 & 8,9 & 8,9 & 8,0 & 8,9 & 8,4 & 8,3 & 6,9 & 7,6 \\
\hline \multirow[t]{3}{*}{ Limburg } & Tot. & 160 & 149 & 308 & 180 & 167 & 347 & $\mathrm{~J} 98$ & 176 & 375 & 208 & 186 & 393 & 224 & 211 & 435 & 194 & 178 & 372 \\
\hline & $\%$ & 13,1 & 12,7 & $|12,9|$ & $|14,5|$ & 13,8 & 14,2 & 15,2 & 13,9 & $|14,6|$ & $|15,6|$ & 14,3 & 14,9 & 15,8 & 15,3 & 15,5 & 14,8 & $|14,0|$ & 14,4 \\
\hline & $\begin{array}{l}\text { Tot. } \\
\text { ":000 }\end{array}$ & $\left|\begin{array}{r}1920 \\
9,9\end{array}\right|$ & 10.4 & $\begin{array}{c}4002 \\
102\end{array}$ & 10,5 & & $\begin{array}{l}4546 \\
10,8\end{array}$ & $\left|\begin{array}{l}2723 \\
12,3\end{array}\right|$ & & & $\begin{array}{l}3264: 3 \\
1.3,8\end{array}$ & 13,9 & & $\left|\begin{array}{l}3845 \\
15,2\end{array}\right|$ & $\left|\begin{array}{l}3792 \\
14,7\end{array}\right|$ & $\left|\begin{array}{l}7637 \\
15,0\end{array}\right|$ & $\left|\begin{array}{r}2789 \\
12,4\end{array}\right|$ & 12,5 & $\begin{array}{r}5659 \\
12,4\end{array}$ \\
\hline
\end{tabular}

Overyssel und Groningen; auch in Zeeland kann eine regelmäßige Zunahme wahrgenommen werden.

Es versteht sich von selbst, daß bei dieser großen Zunahme der Anstaltspatienten verschiedene Faktoren eine große Rolle spielen müssen. Im besonderen richte ich dabei die Aufmerksamkeit auf den Einfluß des Gesetzes zur Regelung der Staatsaufsicht über Irre und Irrenanstalten. Einerseits wirkt dieses Gesetz mehr oder weniger hemmend auf die Behandlung der Geisteskranken in Anstalten, weil es bestimmte Vorschriften für die Aufnahme gibt, woran jeder sich halten muß; hat man irgendwelche Bedenken gegen dieselben, so kann der Patient einfach nicht aufgenommen werden. Andererseits enthält dasselbe Gesetz Bestimmungen über die Pflegekosten, welche der Aufnahme sehr beförderlich sind. Denn als Regel wird doch angenommen, daß im Falle die Angehörigen nicht imstande sind, die Pflegekosten zu bezahlen, die Gemeinde, die Provinz und der Staat dazu beitragen. Es ist begreiflich, daß besonders diese letztere Bestimmung einen großen Einfluß auf die Zunahme der Anstaltspatienten gehabt hat; denn viele, die früher in der Familie versorgt wurden, werden nun in eine Anstalt gebracht, weil die finanziellen Schwierigkeiten aufgehoben sind. 
Es fragt sich nun, ob diese immer wachsende Zunahme kein Ende nehmen wird, da es auf diese Weise zuletzt mehr Geisteskranke als normale Menschen geben würde. Statistischen Angaben und Vergleichungen zufolge würde man zu normalen Verhältnissen kommen, sobald für 10000 Einwohner immer 20 Plätze in einer Anstalt zur Verfügung stünden. Wenn dieses so wäre, müßte also in den Niederlanden noch für 2000 Patienten Platz gemacht werden. Erst dann würde ein Gleichgewicht zustande kommen zwischen denen, welche in eine Anstalt aufgenommen und denen, die entlassen wurden oder starben. Man verweist in dieser Hinsicht nach England, wo allem Anschein nach diese normalen Verhältnisse bestehen.

Endlich noch einiges über die Mittel, die angewendet werden müssen, um den gegenwärtigen Zustand zu verbessern. Niemand wird es bestreiten, daß das Los der Geisteskranken heutzutage viel besser ist al:$f_{t}$ üher; ihr Leben ist jedenfalls ein menschenwürdigeres geworden. Wenngleich in mancher Hinsicht die Verbesserungen, die im Laufe der Jahre zustande kamen, noch nicht zu befriedigenden Resultaten führten, und wir die Zahl der Geheilten gerne größer sehen möchten, wird dennoch niemand die alten \%ustände zurückverlangen. Nein, wir müssen einen anderen Flug nehmen; sowohl, was die hygienischen Zustände als was die Art der Behandlung betrifft, ist alles auch in den Anstalten unseres Landes enorm verbessert, dank der kräftigen Initiative der Staatsbehörde. Die Kenntnis der Art und des Wesens der Psychosen hat aber damit nicht gleichen Schritt gehalten, und wir freuen uns, daß in dieser Zeit zwei neue Kliniken in den Niederlanden errichtet werden, wo das Studium der Psychiatrie ernstlich betrieben werden soll, ebenso wie an den anderen Universitäten in Amsterdam, Leyden und Groningen. Der Professor der Psychiatrie und Neurologie an der Freien Universität in Amsterdam, Prof. L. Bouman, wird dabei von anderem Standpunkt ausgehen als sein Kollege Prof. K. Heilbronner an der Reichsuniversität in Utrecht. Wir hoffen und erwarten, daß die Psychiatrie in unserem Lande von der Arbeit beider Professoren die erwünschten Früchte ernten wird und sich die Resultate in einer größeren Anzahl Geheilter, als bis jetzt beobachtet wurde, zeigen werden.

Eine unbedingte Notwendigkeit dafür ist auch eine Vergrößerung cer ärztlichen Hilfe in den Anstalten. Wenn einem Anstaltsarzt nicht weniger als 150-200 Patienten anvertraut sind, während ihm obendrein oft noch allerlei andere Arbeit auferlegt wird, so bleibt wenig Zeit übrig für die individuelle Behandlung der Patienten. Es ist also wünschenswert, daß die Anzahl der Ärzte in den Anstalten vermehrt und ihre Stellung verbessert wird, damit sie mehr Gelegenheit haben, die verschiedenen Psyehosen sorgfältiger zu prüfen, 
als es bis jetzt möglich war, und sie einem gründlichen Studium zu unterwerfen.

Dadurch würde man das Wesen der Geisteskrankheit besser verstehen lernen und zu einer rationellen Therapie kommen, in der nicht nur physische, sondern auch psychische Faktoren eine wichtige Rolle spielen müssen. 